\title{
Review on Finite Element Method
}

\section{*11ERHUNMWUN, ID; ${ }^{2}$ IKPONMWOSA, UB}

\author{
${ }^{I}$ Department of Production Engineering, University of Benin, Benin City, Nigeria \\ ${ }^{2}$ Department of Petroleum Engineering, University of Benin, Benin City, Nigeria \\ iredia.erhunmwun@uniben.edu benedict.ikponmwosa@uniben.ed $u^{2}$ \\ 08070728898,08162731016
}

\begin{abstract}
In this work, we have discussed what Finite Element Method (FEM) is, its historical development, advantages and its future. The eventual intension of using FEM is to determine the nodal solution of a particular problem under study. The power of FEM is its ability to discretize complex problems and analyse it part by part. Irrespective of the geometry of the problem, with proper mesh refinement, FEM provides very accurate solution. Therefore, FEM is a technique in which a given domain is represented as a collection of simple domains, called finite elements, so that it is possible to systematically construct the approximation functions needed in a variational or weighted-residual approximation of the solution of a problem over each element. (C) JASEM

https://dx.doi.org/10.4314/jasem.v21i5.30
\end{abstract}

Keywords: Weak Formulation, Discretisation, Numerical methods, Finite element method, Global equations, Nodal solution.

One of the numerical methods which can be used for the accurate solution of engineering problems that are either simple or complex is the Finite Element Method. The method was first used in 1956 for aircraft structural problems analysis. Later on, within a decade, the potentialities of the method for the solution of various types of engineering and applied science problems were recognized (Rao, 1982).

The finite element technique has over the years been so well established that today it's seems to be one of the best methods for analysing the efficiency of a wide variety of practical problems. In fact, the method has become one of the research areas for applied mathematicians (Rao, 1982).

The basic idea in the finite element method is actually to find the solution of a complicated problem by replacing it with a simpler one (Rao, 1992).

In recent times, the finite element method involves the use of piecewise continuous functions which is defined over triangular regions. The FEM was first suggested by Courant in 1943 in the literature of applied mathematics (Courant, 1943). Courant's work was ignored until engineers had independently developed it. Early 1906, researchers provided a lattice analogy for stress analysis. This was however replaced by elastic bars of regular pattern. The bar's properties were chosen in a way that caused joints displacements to approximate displacements of the points in the continuum. The method sought to capitalize on the well-known methods of structural analysis (Darrel et al., 2006).

None of the foregoing work was of much practical value at the first time because there were no computers available to generate and solve large set of simultaneous algebraic equations. The development of finite element actually coincided with major advances in digital computers and programming languages.

The search for various methods to discretize continuum mechanics problems has been generally handled with various approaches by mathematicians and engineers. Mathematicians have been able to develop the general methods that are directly applicable to solving the governing set of differential equations. The finite difference method (FDM), residual procedures, are examples of the direct approach and methods of determining the extreme value for some functional. On the other hand, the Engineers have made attempt to solve the problem from a physical point of view by using several types of analogies. McHenry (1943) and Newmark (1949) are examples of this indirect method, that as early as in the 1940's showed that it was possible, with good and acceptable accuracy, to analyse a continuum by removing a piece of the body with an arrangement of trusses. Between 1954 and 1956, Argyris (1950 and 1954) and Turner et al. (1956) were able to show that a more direct substitution of properties could be obtained. These researches were based upon basic assumptions of the behaviour of elements. The term 'finite element' was used for the first time in a presentation by Clough (1960) where the method was presented for a special case of plain stress. After the work by these pioneers, mathematicians and engineers realize that they had been working on the same basic problem but from different approaches. This insight caused a closer agreement between the "analogue" based approach and the "pure" mathematical approach, an agreement which was a basic foundation for further development of the finite element method. For many years now, the finite element method has been considered to be a numerical, and mathematically well defined, discretization method for simulating and analysing a wide variety of boundary value problems. 
Finally, the finite element method is a very versatile method and has found applications in many engineering problems. Today, there are over 100000 engineers that make use of the finite element method (Zienkiewicz, 2000).

The semi-analytical techniques that have found wide application in researches in fluid dynamics were the similarity approach, the perturbation methods, and the integral methods (all for the viscous boundary layer calculations) and the methods of characteristics (for inviscid compressible flow simulations) (Van Dyke, 1964). With respect to the numerical techniques for analysing field problems, Finite Difference based Methods (FDM) were the first to be developed, because of the ease in their implementation (Ray et al., 2010). Despite the fact that the finite difference formulation is relatively simple, the severe limitation faced was that calculations had to be performed manually in the pre-second world war era. Thus, linear problems which involve Laplacian or Biharmonic operators have been solved by relaxation methods iteratively (Richardson, 1910 and Liepman, 1918).

Ritz developed a method in 1909, for structural problems involving elastic deformations, that involves the approximation of the potential functional in terms of trial functions with unknown coefficients (Ritz, 1909). The coefficients which are unknown are determined by minimizing the potential functional. The great limitation of the Ritz method in problem analysis is that the trial functions need to satisfy the boundary conditions of the problem. Courant in 1943 made an outstanding improvement over Ritz method by discretizing the domain of study into triangular areas and assumed a linear trial functions over each of the triangles (Courant, 1943). By this ingenious extension, all the trial functions were not required to satisfy the boundary conditions. Bringing in these methods, the full-fledged development of the FEM was first introduced by Clough (1960). Ever since, the method has made rapid strides for the modelling of structural engineering problems. In recent years, the fluid flow and heat transfer modelling have been accomplished successfully.

The finite element method emerged from the need for analysing complex structural analysis and elasticity problems in civil and aeronautical engineering. Its emergence can be traced to the work by Hrennikoff (1941) and Courant (1943) (Giuseppe, 2007). As stated earlier, the Finite Element Method (FEM) was first developed in 1943 by Courant, who used the Ritz method of numerical analysis and variational calculus minimization to get approximate solutions to vibration systems. Shortly thereafter, Turner et al. (1956) established a broader definition of numerical analysis. The paper centred on the "stiffness and deflection of complex structures".

Numerical solution methods: The various methods of generating numerical solution to problems are:

i. The Rayleigh-Ritz Method

ii. Methods of weighted-residuals

iii. The Petrov - Galerkin method

iv. Least-squares method

v. The Galerkin method

vi. The collocation method

vii. Finite element method

Advantages of using Finite Element Method: The following are some of the advantages of the Finite Element Method over other numerical methods

a. The body analysed can have arbitrary shape, load, and support conditions.

b. It is a very versatile method.

c. The matrix mesh can mix elements of different types.

d. Its versatility can be contained in a single program.

e. The actual structure and its finite element model have a close resemblance.

\section{MATERIALS AND METHODS}

The finite element method is a mathematical tool that is widely used in the analysis of various engineering problems. The procedure is employed extensively in the analysis of solids and structure and of heat transfer and fluids. Finite element method is useful in virtually all fields of engineering analysis. The finite element method is used to solve physical problems in engineering analysis and design. The physical problem typically involves the structure or structural component subjected to certain external loading condition. The idealization of the physical problem to the mathematical model requires certain assumptions that together leads to differential equations governing the mathematical model. Finite element analysis provides an approximate solution to the mathematical model. Since the FEM is a numerical method, it is necessary to assess the solution accuracy. If the accuracy criteria is not met, the numerical solution has to be repeated with refined solution parameters (such as a finer mesh) until a sufficient accuracy is attained. Also, the choice of an approximate mathematical model is crucial and completely determines the insight into the actual physical problem that we can obtain by the analysis.

Finite element method procedure: The step by step procedures for solving a problem using the finite element method are discussed in the following seven major steps. These are: 
Step 1: Establish governing equations and boundary conditions: In order to generate a valid approximate solution to a problem, the differential equation that governs the behaviour and the corresponding boundary conditions for the problem must be determined. This can be done by carrying out mathematical modelling to mimic the behaviour of the problem. Once this is done, the approximate finite element formulation can be used to generate the solution.

Step 2: Discretization of the domain: In this step, the entire solution domain of the problem is subdivided into smaller elements. In doing this, care should be taken to make sure that enough elements are included to capture the behaviour of the solution over the entire domain. Areas of particular interest and care are located where critical values are expected, locations with large gradients, locations where the geometry changes suddenly and locations where boundary conditions and loads are applied. Typically, the larger the number of elements, the smaller the size of the elements and the better the approximation of the solution to the differential equation for a well behaved problem.

Discretization of the given domain into a collection of preselected finite elements involves constructing the finite element mesh of preselected elements, numbering of the nodes and elements and finally generating the geometric properties needed for the problem.

Step 3: Determine the element equations: Once the elements are formed, the algebraic equations to be solved are developed for each individual element. The form of the algebraic equations for every element will be the same. Differences from one element to the next will be due to changes in element size and properties. This is the power of the finite element method. The equations can be written once for a general element. They only need to be modified to reflect a particular element's property and geometry.

Derivation of element equation for all typical elements in the mesh can be formed by constructing the variational formulation of the given differential equation over the typical elements, then, assume that a typical dependent variable $U$ is of the form

$$
U=\sum_{i=1}^{n} U_{i} \psi_{i}
$$

and substitute into the constructed variational formulation of the given differential equation over the typical elements to obtain the element equation in the form

$$
\left[K^{e}\right]\left\{U^{e}\right\}=\left\{f^{e}\right\}
$$

Finally, we derive or select if already available in the literature, element interpolation functions $\psi_{i}$ and compute the equations of the whole problem.

Step 4: Assemble global equations: Once all the element equations are generated, they are put together to form a system of equation for the entire solution domain. In other to do this, we first identify the inter element continuity conditions among the primary variables by relating element nodes to global nodes, then, we identify the equilibrium conditions among the secondary variables and finally we assemble the elements.

Step 5: Imposition of the boundary conditions of the problem: This step helps to reduce the assembled global equations into solvable size. It involves substituting some known values of the parameter under consideration at some known points into the assembled global equations. In other to do this, we must identify the specific primary degrees of freedom.

Step 6: Solution of global equations: The system of equations is solved for the values of the dependent variable at different points on the domain. Depending on the problem type, there may be tens, hundreds, thousands, tens of thousands or even hundreds of thousands of points at which the solution to the differential equation is approximated. This involves computing also the gradient of the solution or other desired quantities from the primary degrees of freedom computed in step 5.

Step 7: Presentation of results: This involves representing the result in tabular and/or graphical form.

Sources of errors in numerical computation: The accuracy of a numerical calculation is quantified by the error of the calculation. Several types of errors can occur in numerical calculations. They include:

1. Error in the parameters of the problem. This is assumed non-existent if they are specified correctly by the analyst.

2. Algebraic errors in the calculations. This type of error is also assumed to be non-existent.

3. Iteration error is the error in an iterative method that approaches the exact solution of the exact problem asymptotically. Iteration errors must decrease towards zero as iteration process progresses. The iteration error itself may be used to determine the successive approximations to the exact solution. Iteration errors can be reduced to the limit of the computing machine. 
4. Approximation error is the difference between the exact solution of an exact problem and the exact solution of an approximation of the exact problem. Approximation errors can be reduced only by choosing a more accurate approximation of the exact problem.

5. Round off error is the error caused by the finite number of digits employed in the calculations. Round off error is more significant when small differences between large numbers are calculated.

\section{RESULTS AND DISCUSSION}

The future of FEM is bright. The application of Finite Element Method is just starting to reach its potential. One of the most exciting prospect is its application to coupled problems like Fluid-structure interaction; thermo-mechanical, thermo-chemical, thermo-chemomechanical problems; bio-mechanics \& bio-medical engineering; piezoelectric, ferroelectric,

electromagnetics etc.

In reality, to simulate nature, we need to be able to solve coupled problems. This is where the exciting problems are today. One of the other exciting areas is in $3 \mathrm{D}$ printing. There has been a huge impetus in the computational mechanics community for simulation of $3 \mathrm{D}$ printing processes. Again, 3D printing is a complex process that involves phase changes, thermal interactions etc. It's again a coupled problem.

There have been many alternative methods proposed in the recent decades. But their commercial applicability is yet to be proved. A recent trend has also been application of cloud-computing for FEM analysis. You can also check out the FEA and CFD software SimScale. It is a cloud-based tool where simulations can be set up on a browser. It does not need the hassle of licenses or installations. It allows structural, fluid and particle simulations. SimScale offers a free version where one can get up to 3000 hours for computing. For the free version, the projects created are publicly available. However, if one would like to keep your project private, a professional version is also available. You can explore the public projects database, where there are loads of projects already available and could find some interesting ideas on new topics.

In short, FEM is just starting to make an impact on the radar! There are great potentials and promises for the coming decades!

Conclusion: In this research, we have looked at the history of the Finite Element Method, the advantages of using FEM, the various steps involved in FEM, the sources of errors in numerical computation and finally the future of FEM. It is highly rewarding to study and employ FEM in solving problem. Today, virtually everything can be discretised and divided into finite element whether it be solid, liquid or gas. Therefore, all countries in the world must be made to realise that their most needful tool of change lies in their proper use of the Finite Element Method.

\section{REFERENCES}

Argyris, J (1950, 1954). Energy Theorems and Structural Analysis. Aircraft Engineering. In 1960 these papers were consolidated in a book by Butterworths Scientific Publications titled Energy Theorems and Structural Analysis.

Clough, RW (1960). The Finite Element Method in Plane Stress Analysis. Proc. $2^{\text {nd }}$ ASCE Conf. On Electronic Computation, Pittsburg, $\mathrm{Pa}$

Courant, R (1943). Variational Methods for the Solution of Problems of Equilibrium and Vibration. Bulletin of the American Mathematical Society, 49, pp. 1-23.

Darrel, W; Pepper and Juan C; Heinrich (2006). The Finite Element Method, Taylor and francis, New York.

Giuseppe, P (2007). The finite-element method, Part I: R. L. Courant: Historical Corner.

Hrennikoff, A (1941). Solution of Problems in Elasticity by the framework Method. J. Appl. Mech. Vol. 8, pp. 169-175.

Liepman, H (1918). Acad. Wiss., Math. Phys. Klasae, Sitzungsber, vol. 3 , pp. 388 .

McHenry, D (1943). A Lattice Analogy for the Solution of Plane Stress Problems. J. Inst. Civil Eng. 21(2), pp. 59-82.

Newmark, NM (1949). Numerical methods of analysis in bars, plates and elastic bodies. In L. E. Grinter, editor, Numerical Methods in Analysis in Engineering. Macmillan, New York.

Rao, SS (1982). The Finite Element Method in Engineering, Second Edition, Pergamon Press, Oxford, U.K.

Rao, SS (1992). Finite Element Method in Engineering. Second and Third edition

Ray, B; Biswas, G; Sharma, A (2010). Generation of secondary droplets in coalescence of a drop at a liquid/ liquid interface. J. of Fluid Mechanics, 655, pp. 72-104.

Richardson, LF (1910). Phil. Trans. The Royal Society of London, Ser-A, 210, pp. 307-357.

Ritz, W. (1909). Zeitschrift fur Angewandte Mathematik and Mechanik, 35, pp. 1-61.

Turner, M; Clough, RW; Martins, HC; Topp, LJ (1956). Stiffness and Deflection Analysis of Complex Structures. J. Aeronautical Science, 23(9), pp. 805823.

Van Dyke, M (1964). Perturbation Methods in Fluid Mechanics. Academic Press.

Zienkiewicz, OC (2000). The Finite Element Method, $5^{\text {th }}$ edition (volume 1) The Basic. 